Original Full Length Article

\title{
Improved bone defect healing by a superagonistic GDF5 variant derived from a patient with multiple synostoses syndrome
}

\author{
Elisa Degenkolbe a,b,1 ${ }^{\text {, Carolin Schwarz }}{ }^{\text {a,b,c,1 }}$, Claus-Eric Ott ${ }^{\text {d,e }}$, Jana König ${ }^{\text {a,b,2 }}$, Katharina Schmidt-Bleek ${ }^{\mathrm{c}}$, \\ Agnes Ellinghaus ${ }^{c}$, Tanja Schmidt ${ }^{c}$, Jasmin Lienau ${ }^{c, 3}$, Frank Plöger ${ }^{f}$, Stefan Mundlos ${ }^{\text {a,d,e }}$, Georg N. Duda ${ }^{\text {a,c }}$, \\ Bettina M. Willie ${ }^{c}$, Petra Seemann a,b,e,*
}

\footnotetext{
a Berlin-Brandenburg Center for Regenerative Therapies (BCRT), Charité - Universitätsmedizin Berlin, 13353 Berlin, Germany

b Berlin-Brandenburg School for Regenerative Therapies (BSRT), Charité - Universitätsmedizin Berlin, 13353 Berlin, Germany

c Julius Wolff Institute and Center for Musculoskeletal Surgery, Charité - Universitätsmedizin Berlin, 13353 Berlin, Germany

d Institute for Medical Genetics and Human Genetics, Charité - Universitätsmedizin Berlin, 13353 Berlin, Germany

e Research Group Development and Disease, Max Planck Institute for Molecular Genetics, 14195 Berlin, Germany

${ }^{\mathrm{f}}$ Biopharm GmbH, 69115 Heidelberg, Germany
}

\section{A R T I C L E I N F O}

\section{Article history:}

Received 16 October 2014

Revised 16 December 2014

Accepted 17 December 2014

Available online 24 December 2014

Edited by Michael Amling

\section{Keywords:}

Growth and Differentiation Factor 5

NOGGIN

Bone Morphogenetic Proteins

Bone healing

Inflammation

Endochondral bone formation

\begin{abstract}
A B S T R A C T
Multiple synostoses syndrome 2 (SYNS2) is a rare genetic disease characterized by multiple fusions of the joints of the extremities, like phalangeal joints, carpal and tarsal joints or the knee and elbows. SYNS2 is caused by point mutations in the Growth and Differentiation Factor 5 (GDF5), which plays an essential role during skeletal development and regeneration. We selected one of the SYNS2-causing GDF5 mutations, p.N445T, which is known to destabilize the interaction with the Bone Morphogenetic Protein (BMP) antagonist NOGGIN (NOG), in order to generate the superagonistic GDF5 variant GDF5 ${ }^{\mathrm{N} 45 \mathrm{~T}}$. In this study, we tested its capacity to support regeneration in a rat critical-sized defect model in vivo. MicroCT and histological analyses indicate that GDF5 ${ }^{\mathrm{N} 445 \mathrm{~T}}$-treated defects show faster and more efficient healing compared to GDF5 wild type (GDF5 ${ }^{\mathrm{wt}}$ )-treated defects. Microarraybased gene expression and quantitative PCR analyses from callus tissue point to a specific acceleration of the early phases of bone healing, comprising the inflammation and chondrogenesis phase. These results support the concept that disease-deduced growth factor variants are promising lead structures for novel therapeutics with improved clinical activities.
\end{abstract}

(C) 2014 Elsevier Inc. All rights reserved.

\section{Introduction}

Bone growth and restoration comprise a well-orchestrated interplay of various factors and mediators responsible for cellular recruitment, proliferation and differentiation. Secreted growth factors, in particular Bone Morphogenetic Proteins (BMPs) and Growth and Differentiation Factors (GDFs), play a key role as they exert their effects on mesenchymal stem cells by promoting their differentiation into the chondro- or osteogenic lineage [1,2].

GDF5 is known as BMP14 or Cartilage-Derived Morphogenetic Protein 1 (CDMP1). It is a central modulator of early cartilage formation and plays an important role in the development of bones, joints,

\footnotetext{
* Corresponding author at: Berlin-Brandenburg Center for Regenerative Therapies, Charité - Universitätsmedizin Berlin, Augustenburger Platz 1, 13353 Berlin, Germany. Fax: + 49304507539436 .

E-mail address: petra.seemann@charite.de (P. Seemann).

${ }^{1}$ Shared first authorship.

2 Present affiliation: Roche Diagnostics International AG, 6343 Rotkreuz, Switzerland.

${ }^{3}$ Present affiliation: Department of Internal Medicine/Infectious Diseases and Pulmonary Medicine, Charité-Universitätsmedizin Berlin, 13353 Berlin, Germany.
}

tendons and ligaments in the axial and appendicular skeleton [3-6]. As a therapeutic agent, the regeneration-supporting activities of wild type GDF5 (GDF5 ${ }^{\mathrm{wt}}$ ) have been investigated in various preclinical studies and clinical trials including craniofacial, peri-implant $[7,8]$ and calvarial $[9,10]$ bone formation, spine $[11-13]$ and long bone surgery $[14,15]$ as well as cartilage [16], tendon and ligament [17-19] formation and repair [20].

GDF5 is also implicated in rare diseases associated with bone overgrowth, like the multiple synostoses syndrome (SYNS2, \#610017). SYNS2 is characterized by fusion of carpal and tarsal bones as well as proximal symphalangism in fingers and toes [21-24]. Previous work analyzing the signaling pathomechanism behind the SYNS2-associated GDF5 mutations p.W414R [25], p.S475N [22] and p.N445T [23] revealed that the mutations abrogate the negative feedback loop controlling GDF5 activity by extracellular antagonists like NOGGIN (NOG). Most of the aforementioned GDF5 mutations interfere with NOG binding and affect GDF5 receptor specificity due to their position within the overlapping receptor and NOG binding domain. For example, GDF5 ${ }^{\mathrm{W} 414 \mathrm{R}}$ is incapable of BMP receptor type 1A (BMPR1A) binding and also its binding to BMPR1B is diminished [25]. Similarly, GDF5 ${ }^{\text {S475N }}$ shows only 
weak binding to BMPR2 [22]. In contrast, GDF5 ${ }^{\mathrm{N} 445 \mathrm{~T}}$ displays unaltered receptor binding characteristics as demonstrated in a previous in vitro study [23]. The profound NOG insensitivity of GDF5 ${ }^{\mathrm{N} 445 \mathrm{~T}}$ increases its biological activity compared to the wild type counterpart as shown in a chondrogenic differentiation assay [23].

The motivation behind this study was an attempt to learn from rare diseases for designing biomolecules with improved biological activities and therapeutic properties. We hypothesized that the naturally occurring superagonistic GDF5 ${ }^{\mathrm{N} 445 \mathrm{~T}}$ molecule accelerates and improves the healing process when compared with its wild type counterpart in a critical-sized bone defect model.

\section{Materials \& methods}

\section{Recombinant proteins}

Recombinant human GDF5 ${ }^{\mathrm{wt}}$ and its variant GDF5 ${ }^{\mathrm{N} 445 \mathrm{~T}}$ were provided by Biopharm $\mathrm{GmbH}$ and dissolved in $10 \mathrm{mM} \mathrm{HCl}$.

\section{Animal surgery}

A group of 72 female Sprague-Dawley rats (12-week-old, weight 250-300 g; Charles River Deutschland GmbH, Germany) underwent a $5 \mathrm{~mm}$ critical-sized femoral segmental defect followed by stabilization with a custom-made unilateral external fixator as described previously $[26,27]$. The rats were administered an intraperitoneal injection of a solution containing ketamine hydrochloride $(60 \mathrm{mg} / \mathrm{kg}$, ketamin $50 \mathrm{mg}$, Actavis ${ }^{\circledR}$, Island) and medetomidine $\left(0.3 \mathrm{mg} / \mathrm{kg}\right.$, Domitor ${ }^{\circledR}$, Pfizer, Karlsruhe). The antibiotic clindamycin-2-dihydrogenphosphate (45 mg/kg, Ratiopharm, Ulm) was also administered subcutaneously. An incision was made across the lateral aspect of the thigh, through the fascia, exposing the femur by separating the gluteus superficialis and biceps femoralis muscles. The fixation device consisted of 4 titanium-threaded pins ( $0.65 \mathrm{~mm}$ core diameter $/ 1.2 \mathrm{~mm}$ outer diameter; Medizintechnik Jagel, Germany) held between the two sides of the stainless steel crossbar (dimension $22 \times 5 \times 2 \mathrm{~mm}$ ) under compression. Four threaded titanium pins were manually screwed into the femur after pre-drilling with a $0.9 \mathrm{~mm}$ drill bit resulting in a firm fixation. The offset distance, free length of the pins between the rats' lateral femoral surface and the inner side of the fixator bar, was $7.5 \mathrm{~mm}$. In vitro mechanical testing indicated that the fixator construct had an axial stiffness of $62.02 \pm 13.52 \mathrm{~N} / \mathrm{mm}$ (mean $\pm \mathrm{SD}$ ) and torsional stiffness of $15.35 \pm 2.69 \mathrm{~N} / \mathrm{mm}[26]$.

Following generation of the $5 \mathrm{~mm}$ femoral defect in the middle of the femur using an oscillating saw by performing a double transverse osteotomy, absorbable type I bovine collagen sponges $(10 \times 5 \times$ $5 \mathrm{~mm}$; Lyostypt, B. Braun, Germany) were implanted within the osteotomy gap. The rats were randomly subdivided into two experimental groups and one control group. For the two experimental groups, the sponges were loaded either with $50 \mu \mathrm{g}$ GDF5 ${ }^{\mathrm{wt}}$ or $50 \mu \mathrm{g}$ GDF5 ${ }^{\mathrm{N} 445 \mathrm{~T}}$, dissolved in resuspension buffer $(10 \mathrm{mM} \mathrm{HCl})$, and placed into the defect. The dose of $50 \mu \mathrm{g}$ per defect was calculated for a $300 \mathrm{~g}$ body weight of a rat based on the dose used in the clinics (12 mg for BMP2) for patients with an average body weight of $75 \mathrm{~kg}$. The control group was treated with collagen sponges soaked in resuspension buffer. The fascia and skin were sutured and the analgesic tramadol hydrochloride was administered subcutaneously during the surgery $(20 \mathrm{mg} / \mathrm{kg}$, Grünenthal, Aachen) and over 3 days diluted in the animals' drinking water (25 mg/L, Grünenthal, Aachen). Immediately after surgery, the rats were allowed to resume normal activity and given unrestricted access to food and water. For analysis of the early healing phase, 48 rats ( $n=4$ rats/group \& time point) were sacrificed at days 3, 5, 7 and 14 post-operation and the callus tissue between the two inner pins was harvested for RNA extraction and gene expression analyses. A further 24 rats ( $n=8$ rats/group \& time point) were analyzed by in vivo micro-computed tomography (microcT) at 2, 4 and 6 weeks post- operation, to assess the overall regenerative capacity of the growth factor-treated defects and the control group. Most of the animals (90.3\%) tolerated the experimental procedure well and had an uncomplicated recovery through the end of the study; however, the following complications were observed: anesthesia-associated death $(n=5)$, pin infection $(n=1)$, pin breakage $(n=1)$. The anesthesia-associated deaths occurred in 3 rats directly following osteotomy surgery, while the other 2 rats died under anesthesia during the first in vivo microCT scan. After exclusion of these 7 rats, the remaining 17 rats, which had no complications, completed the in vivo microCT assessment $(n=5-6$ rats/group \& time point). These rats were sacrificed after 6 weeks of healing and histomorphometric analysis was performed $(n=4-6$ rats/group). The osteotomized femur from 2 rats was excluded from histomorphometric analysis due to improper sectioning. All animal experiments were carried out according to the policies and procedures established by the Animal Welfare Act, the NIH Guide for Care and Use of Laboratory Animals, and the National Animal Welfare Guidelines. The study was approved by the local authorities (LaGeSo Berlin, G0210/ 08, G0071/07).

\section{Micro-computed tomography}

Bone defect healing ( $n=5-6 /$ group) was assessed by in vivo microCT (vivaCT 40, Scanco Medical, 55 kVp, $145 \mu \mathrm{A}, 150 \mathrm{~ms}$ integration time) at 2, 4 and 6 weeks post-operation. Analysis was performed on the basis of published procedures [28-30] using the specific parameters described below. We used a semi-automated segmentation of crosssectional tomograms to derive the volume of interest (VOI), defined by the periosteal callus as the outer boundary and the endosteal callus as the inner boundary, excluding the original (parent) cortical bone. The VOI included the $5 \mathrm{~mm}$ defect region and $0.5 \mathrm{~mm}$ in the proximal and distal directions from the borders of the original osteotomy. A global threshold of $50 \%$ of the mineral density of the intact limb, equivalent to $351 \mathrm{mg} \mathrm{HA} / \mathrm{cm}^{3}$ was used to distinguish mineralized tissue (bone and calcified cartilage) from non-mineralized tissue. Outcome measures included mineralized callus volume (bone volume, $\mathrm{BV}, \mathrm{mm}^{3}$ ), total callus volume $\left(\mathrm{TV}, \mathrm{mm}^{3}\right)$, mineralized callus volume fraction $\left(\mathrm{BV} / \mathrm{TV}, \mathrm{mm}^{3} /\right.$ $\mathrm{mm}^{3}$ ) and tissue mineral content (TMC, mg HA), defined as BV multiplied by the total mineral density (TMD). TMD was calculated using only the voxels whose intensity exceeded the threshold. In addition, the number of animals achieving bony defect bridging in the defect area was quantified. For microCT data, normal distribution was tested using a Shapiro-Wilk test and the within-subject effect of healing time $(2,4$, and 6 weeks post-operation) and treatment (control, $\mathrm{GDF}^{\mathrm{wt}}$, GDF5 ${ }^{\mathrm{N} 445 \mathrm{~T}}$ ) were assessed using a repeated measures ANOVA. Sub-analyses were also performed using paired or unpaired t-tests, as appropriate. All values are presented as mean \pm SD and statistical significance was set at $p<0.05$. Analyses were performed using SAS 9.3 software.

\section{Qualitative and quantitative histology}

After in vivo microCT analysis 6 weeks post-operation, rats were sacrificed and femora were harvested for histological and histomorphometric analyses ( $n=4-6$ /group). Femora were fixed in formaldehyde for $48 \mathrm{~h}$ and subsequently decalcified in EDTA for approximately 4 weeks at $37^{\circ} \mathrm{C}$. Fixed and decalcified tissues were dehydrated in graded ethanol up to $100 \%$, transferred to xylene, and embedded in paraffin. Four micron thick longitudinal sections were prepared on a customary microtome (Leica RM 2125, Germany) and placed on glass slides. Quantitative histomorphometry was performed to analyze tissue differentiation for a single fixed region of interest (ROI), using semiautomated software (KS400 3.0 software, Zeiss MicroImaging GmbH, Germany). The composition of the callus tissue was quantified after staining with Movat Pentachrome by measuring the area within the osteotomy gap occupied by bone (yellow), cartilage (blue to green), 
and fibrous connective tissue formation (pink to purple), with the fibrous connective tissue also including bone marrow elements. Tissue areas $\left(\mathrm{mm}^{2}\right)$ were measured and tissue fractions (\%) calculated based on the ROI. For histomorphometry data, normal distribution was tested using a Shapiro-Wilk test and a one-way ANOVA was performed to assess the effect of treatment (control, GDF5 ${ }^{\mathrm{wt}}$, GDF5 ${ }^{\mathrm{N} 445 \mathrm{~T}}$ ). Sub-analyses were also performed using unpaired t-tests. All values are presented as mean \pm SD and statistical significance was set at $p<0.05$. Analyses were performed using SAS 9.3 software.

\section{RNA isolation}

Rat callus tissue between the two inner pins was harvested at days $3,5,7$ and 14 post-operation ( $n=4$ rats/group \& time point) and immediately flash frozen in liquid nitrogen. Rat tissue was subsequently pulverized in pre-cooled Teflon grinding jars (Retsch $\mathrm{GmbH}$, Germany). Total RNA was isolated using Trifast reagent (Peqlab, Germany) and RNeasy Mini Kit (Qiagen, Germany) according to the manufacturer's instructions. RNA concentrations were determined spectrophotometrically and RNA integrity was verified by denaturing RNA gel electrophoresis. The isolated total RNA was stored at $-80{ }^{\circ} \mathrm{C}$.

\section{Quantitative PCR (Q-PCR)}

Using TaqMan® Reverse Transcription Reagents (Applied Biosystems, CA), cDNA was synthesized from $1 \mu \mathrm{g}$ of RNA. Q-PCR was performed in MicroAmp optical 384-well plates (Applied Biosystems, CA) on an ABI Prism 7900 Sequence Detection System (Applied Biosystems, C). As detection reagent, the SensiFast ${ }^{\text {TM }}$ SYBR Hi-ROX Kit (Bioline, Germany) was used. Primers for target genes were designed to span exon-exon junctions (Table S1). Samples were run in triplicates and results were normalized to Sfrs3 or $18 \mathrm{~S} r R N A$ using the $\Delta \Delta \mathrm{C}_{\mathrm{t}}$ method. Graphs display the fold change of transcript concentrations in each experimental group in comparison to the control at the earliest time point.

Q-PCR analyses of the individual rat RNAs showed comparable gene expression patterns at days 3, 7 and 14 (Fig. S1). For gene expression analyses by Q-PCR and microarray hybridizations, individual RNA samples of each experimental group (control, GDF5 ${ }^{\mathrm{wt}}$, GDF5 $^{\mathrm{N} 445 \mathrm{~T}}$ at days $3,5,7$ and 14 ) were pooled. According to the quality of the isolated RNA, three samples per group were selected and pooled for these comparisons.

Using the D'Agostino-Pearson omnibus normality test we confirmed that Q-PCR results of individual animals were normally distributed. Dependent on the data structure, we applied either a one-way ANOVA with Sidak post-hoc test or a two-tailed Student's $t$-test for statistical analyses. Analyses were performed using GraphPad Prism 6 software.

\section{Microarray analysis}

The pooled RNA samples of each experimental group (control, $\mathrm{GDF}^{\mathrm{wt}}$, and GDF5 ${ }^{\mathrm{N} 445 \mathrm{~T}}$ at time points days 3, 5, 7 and 14) were analyzed at the Laboratory of Functional Genomics Charité (LFGC) using the Affymetrix GeneChip expression analysis system (GeneChip Rat Gene 1.0 ST Array; Affymetrix, CA). Microarray data are available in the ArrayExpress database (www.ebi.ac.uk/arrayexpress) under the accession number E-MTAB-2249.

Data pre-processing was performed using the 'aroma affymetrix' package (version 2.9.4) which is implemented in the $\mathrm{R}$ software (http://www.r-project.org) [31]. In order to get gene-level summaries for each gene, default settings were used, i.e. RMA background correction, quantile normalization, and probe-level summarization (plm). Gene-level summarization on log2-scale was annotated to gene symbols using the 'ragene10sttranscriptcluster.db' package (version 7.0.1). The 'arrayQualityMetrics' package (version 3.14.0) was used for quality metrics of raw data and the obtained expression set [32]. Statistical analysis was conducted based on variance over a time course of 4 measurements (day 3 , day 5 , day 7 , and day 14 ). Variances were calculated for each experimental group, i.e. control, GDF5 ${ }^{\mathrm{wt}}$, and $\mathrm{GDF}^{\mathrm{N} 445 \mathrm{~T}}$. From each group, the top $1.5 \%$ of genes with the highest variance were selected. Taken together, this resulted in a total of 517 high-variance genes. For clustering analysis of the different samples according to similarity in pattern of gene expression, expression values of each of the 517 high-variance genes were scaled to a mean of zero and a standard deviation of one. For the heat plot, scaling was performed separately for each experimental group, i.e. control, GDF5 ${ }^{\mathrm{wt}}$, and GDF5 ${ }^{\mathrm{N} 445 \mathrm{~T}}$. The normalized values of 4 time points per group were then combined into vectors of 12 values per gene and hierarchical clustering based on these vectors was performed to group genes according to similarity in pattern of gene expression. In the line plots, summary values for the different clusters were plotted as mean \pm SD after scaling all values per gene, thus allowing estimation of differences of expression levels dependent on different treatments. The lines connect the 4 time points analyzed in each group. Model-based gene set Gene Ontology (GO) analysis [33] was performed for all 517 high-variance genes, whereby GO terms with a marginal probability of at least $50 \%$ were considered as significant. Additionally, the Parent-Child-Union method of the Ontologizer [34,35] was used to analyze each cluster separately, and GO terms with Benjamini-Hochberg corrected $\mathrm{p} \leq 0.05$ were considered as significant.

\section{Results}

\section{Enhanced bone defect healing by GDF5 ${ }^{N 445 T}$ compared to GDF5 ${ }^{w t}$}

The growth factor variant GDF5 ${ }^{\mathrm{N} 445 \mathrm{~T}}$ was locally applied into a rat critical-sized femoral segmental defect model. The healing potential of GDF5 $^{\mathrm{N} 445 \mathrm{~T}}$ was assessed by in vivo microCT at weeks 2, 4, and 6 and histomorphometry at 6 weeks post-operation (Fig. 1).

In vivo microCT analysis revealed moderate mineralized tissue formation around the cortical ends of the defect site at week 2 for both the GDF5 ${ }^{\mathrm{N} 445 \mathrm{~T}}$ - and the GDF5 ${ }^{\mathrm{wt}}$-treated defects. Periosteal, intracortical and endosteal bony bridging with marrow elements and early restoration of the medullary canal were observed in the GDF5 $^{\text {N445T }}$ group at weeks 4 (5/6 animals) and 6 (6/6 animals) with cartilage islands and minimal remnants of mineralized cartilage in the defect region, respectively. Within the GDF5 ${ }^{\text {wt }}$ group endosteal bridging was achieved by $2 / 6$ animals at week 4 and by $4 / 6$ animals at week 6 with one animal already achieving intracortical bridging at week 6 post-osteotomy. GDF5 ${ }^{\mathrm{wt}}$-treated defects showed cartilage tissue and endochondral bone formation at the endosteal defect site with thick fibrous connective tissue fibers separating muscle tissue from the gap (Fig. 1).

Mineralized callus volume (BV) measured by microCT increased from the 4 th to the 6 th week post-osteotomy in both the GDF5 ${ }^{\mathrm{wt}}$ and the GDF5 ${ }^{\mathrm{N} 445 \mathrm{~T}}$-treated animals. In both groups, the mineralized callus tissue was primarily concentrated within the endosteal region rather than in the periosteal region, thus resulting in diminished bone marrow space over time. The GDF5 ${ }^{\mathrm{N} 445 \mathrm{~T}}$ group showed significantly greater mineralized callus volume (BV, $p<0.01$ ), total callus volume (TV, $p<0.01$ ), and total tissue mineral content (TMC, $p<0.01$ ) as compared to the GDF5 ${ }^{\text {wt }}$-treated defects (Table 1 ). Furthermore, histomorphometry at week 6 demonstrated a significantly greater bone area for GDF5 ${ }^{\mathrm{N} 445}$ (BAr, $p=0.01$ ) as compared to GDF5 $^{\text {wt }}$-treated defects (Table 2).

The defects of the control group all resulted in a non-union at 6 weeks post-osteotomy. The empty defects were characterized by fibrous connective tissue and/or prolapsed muscle tissue as well as rounded cortical bone ends and newly formed bone between the cortices of each fragment, with sealing of the medullary canal from the osteotomy. 

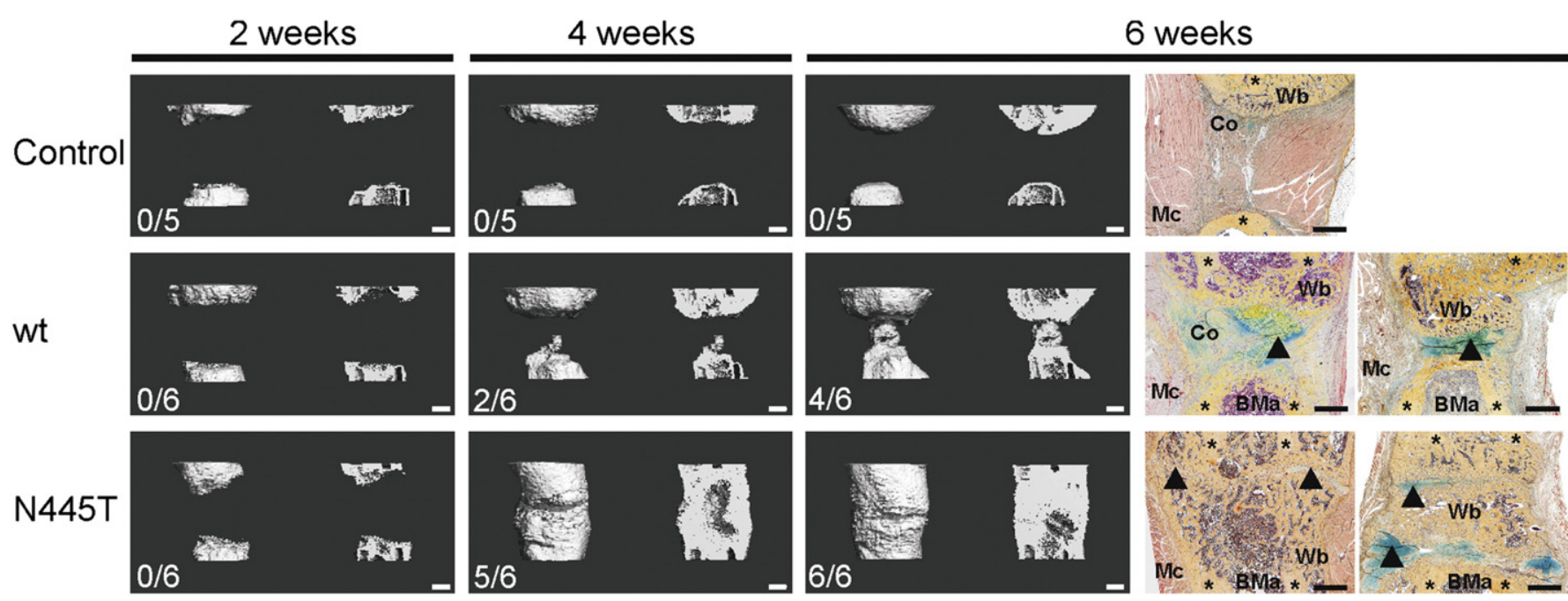

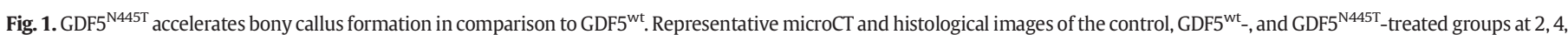

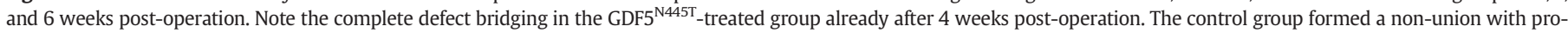

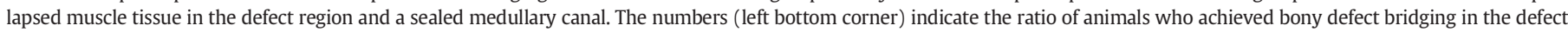

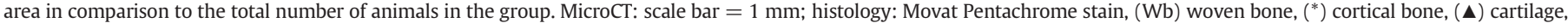
(BMa) bone marrow, (Co) fibrous connective tissue, (Mc) muscle tissue, scale bar $=1 \mathrm{~mm}$.

\section{Transcriptional feedback response after GDF5 treatment}

To identify a potential negative feedback loop in direct response to the growth factor treatment, we analyzed the transcriptomes after GDF5 treatment on Gdf5 itself and a number of known extracellular modulators of GDF5 signaling at the earliest time point analyzed, i.e., day 3 after osteotomy (Figs. 2 and S2).

In response to the GDF5 treatment, expression of Gdf5 is not altered 3 days post-stimulation. The expression of most of the BMP antagonists (Fig. S2) remained also unaffected by the treatments with the notable exceptions of Nog and Chrdl2 (Fig. 2). The up-regulation of Nog likely diminishes GDF5 ${ }^{\text {wt }}$ action without affecting GDF5 ${ }^{\text {N445T }}$ activity, as GDF5 $^{\text {N445T }}$ is NOG insensitive. In contrast, we neither observed

Table 1

Summary of in vivo microCT data for all experimental groups at weeks 2, 4 and 6 postoperation.

\begin{tabular}{|c|c|c|c|}
\hline Parameter/Group & Control $\mathrm{n}=5$ & $\mathrm{GDF}^{\mathrm{wt}} \mathrm{n}=6$ & $\mathrm{GDF}^{\mathrm{N} 445 \mathrm{~T}} \mathrm{n}=6$ \\
\hline \multicolumn{4}{|l|}{$\mathrm{BV} / \mathrm{TV}[\%]^{\mathrm{a}, \mathrm{b}}$} \\
\hline 2 weeks & $0.6 \pm 0.1$ & $0.6 \pm 0.1$ & $0.6 \pm 0.1$ \\
\hline 4 weeks & $0.6 \pm 0.1$ & $0.7 \pm 0.1$ & $0.8 \pm 0.1$ \\
\hline 6 weeks & $0.7 \pm 0.1$ & $0.8 \pm 0.1$ & $0.8 \pm 0.0$ \\
\hline \multicolumn{4}{|l|}{$\mathrm{BV}\left[\mathrm{mm}^{3}\right]^{\mathrm{a}, \mathrm{b}}$} \\
\hline 2 weeks & $10.6 \pm 4.3$ & $13.6 \pm 3.0$ & $16.3 \pm 6.1$ \\
\hline 4 weeks $^{c}$ & $13.2 \pm 3.1$ & $33.0 \pm 12.2$ & $76.5 \pm 25.3$ \\
\hline 6 weeks $^{\mathrm{c}}$ & $22.7 \pm 10.5$ & $53.8 \pm 18.5$ & $109.3 \pm 25.5$ \\
\hline \multicolumn{4}{|l|}{$\mathrm{TV}\left[\mathrm{mm}^{3}\right]^{\mathrm{a}, \mathrm{b}}$} \\
\hline 2 weeks & $16.1 \pm 5.3$ & $21.5 \pm 4.8$ & $27.1 \pm 11.1$ \\
\hline 4 weeks $^{c}$ & $20.7 \pm 4.8$ & $47.8 \pm 14.4$ & $99.7 \pm 25.6$ \\
\hline 6 weeks $^{c}$ & $31.5 \pm 15.8$ & $67.4 \pm 19.8$ & $131.1 \pm 32.1$ \\
\hline \multicolumn{4}{|l|}{$\mathrm{TMC}[\mathrm{mg} \mathrm{HA}]^{\mathrm{a}, \mathrm{b}}$} \\
\hline 2 weeks & $6.7 \pm 2.8$ & $7.8 \pm 1.5$ & $9.2 \pm 3.3$ \\
\hline 4 weeks $^{\mathrm{c}}$ & $8.5 \pm 1.6$ & $21.1 \pm 8.4$ & $47.1 \pm 16.4$ \\
\hline 6 weeks $^{c}$ & $15.7 \pm 6.5$ & $36.9 \pm 13.4$ & $75.0 \pm 17.8$ \\
\hline \multicolumn{4}{|c|}{ TMD $\left[\mathrm{mg} \mathrm{HA} / \mathrm{cm}^{3}\right]^{\mathrm{a}, \mathrm{b}}$} \\
\hline 2 weeks & $626.7 \pm 54.0$ & $574.0 \pm 16.3$ & $567.2 \pm 18.7$ \\
\hline 4 weeks $^{c}$ & $646.9 \pm 38.4$ & $636.4 \pm 18.6$ & $612.5 \pm 13.2$ \\
\hline 6 weeks & $699.6 \pm 36.5$ & $683.7 \pm 32.7$ & $685.8 \pm 22.9$ \\
\hline
\end{tabular}

a Between-subject effects of treatment.

b Within-subject effects of healing time, ANOVA, $\mathrm{p}<0.05$.

$\mathrm{GDF}^{\mathrm{wt}}$ different from GDF5 ${ }^{\mathrm{N} 445 \mathrm{~T}}$, unpaired $t$-test $\mathrm{p}<0.05$; BV mineralized callus

volume,TV total callus volume, BV/TV mineralized callus volume fraction, TMC tissue mineral content, TMD tissue mineral density, HA hydroxyapatite; data is given as mean $\pm \mathrm{SD}$. inhibition of GDF5 ${ }^{\mathrm{wt}}$ nor by GDF5 ${ }^{\mathrm{N} 445 \mathrm{~T}}$ by CHRDL2 (Fig. S3). Therefore, the altered NOG-interaction may contribute to the faster healing process observed for GDF5 ${ }^{\mathrm{N} 445 \mathrm{~T}}$.

\section{GDF5 ${ }^{\text {N445T }}$ application causes distinct effects on the gene expression pattern}

In order to explore and compare the early molecular changes in response to the local growth factor applications, we performed global gene expression profiling. A total of 517 genes representing the top $1.5 \%$ of high-variance genes derived from each experimental group were included into further analyses. Unsupervised hierarchical clustering was used to visualize the overall similarity of gene expression patterns in all samples. Distinct gene expression patterns can be identified already 3 or 5 days after GDF5 ${ }^{\mathrm{wt}}$ and GDF5 ${ }^{\mathrm{N} 445 \mathrm{~T}}$ application, respectively. The largest differences between the treatment groups are present 3 days after growth factor application. Notably, the gene expression patterns diverge at days 7 and 14, when the GDF5 ${ }^{\mathrm{N} 445 \mathrm{~T}}$-treated samples branch away from control and GDF5 ${ }^{\mathrm{wt}}$-treated samples, indicating qualitatively different treatment effects of the growth factors (Fig. S4). GO analysis of all 517 high-variance genes revealed 'ossification' as the GO term with the highest marginal followed by GO terms such as 'muscle contraction' and 'cell chemotaxis' which are highly relevant processes in skeletal biology and fracture healing. To further explore gene expression in the time course of the healing process, genes were clustered according to similarity of their expression pattern and visualized by a

Table 2

Data from the histomorphometric analysis for all experimental groups at week 6 postoperation.

\begin{tabular}{lccc}
\hline Region/Group & Control $\mathrm{n}=4$ & GDF5 $^{\mathrm{wt}} \mathrm{n}=6$ & GDF5 $^{\mathrm{N} 445 \mathrm{~T}} \mathrm{n}=5$ \\
\hline Total area $\left[\mathrm{mm}^{2}\right]$ & $18.9 \pm 9.3$ & $25.5 \pm 10.9$ & $28.0 \pm 3.5$ \\
Bone area $\left[\mathrm{mm}^{2}\right]^{\mathrm{a}, \mathrm{b}}$ & $3.2 \pm 2.3$ & $8.2 \pm 2.9$ & $12.9 \pm 2.0$ \\
Cartilage area $\left[\mathrm{mm}^{2}\right]^{\mathrm{a}}$ & $0.1 \pm 0.1$ & $1.6 \pm 1.0$ & $2.0 \pm 1.6$ \\
$\begin{array}{l}\text { Fibrous connective tissue } \\
\quad \text { area }\left[\mathrm{mm}^{2}\right]\end{array}$ & $13.2 \pm 8.3$ & $15.5 \pm 8.0$ & $13.1 \pm 1.5$ \\
Muscle area $\left[\mathrm{mm}^{2}\right]^{\mathrm{a}}$ & $2.4 \pm 2.3$ & $0.3 \pm 0.5$ & $0.0 \pm 0.0$ \\
\hline
\end{tabular}

a Between-subject effects of treatment, ANOVA, $\mathrm{p}<0.05$,

b GDF5 $^{\mathrm{wt}}$ different from GDF5 ${ }^{\mathrm{N} 44 \mathrm{~T}}$, unpaired $t$-test $\mathrm{p}<0.05$; data is given as mean $\pm \mathrm{SD}$. 

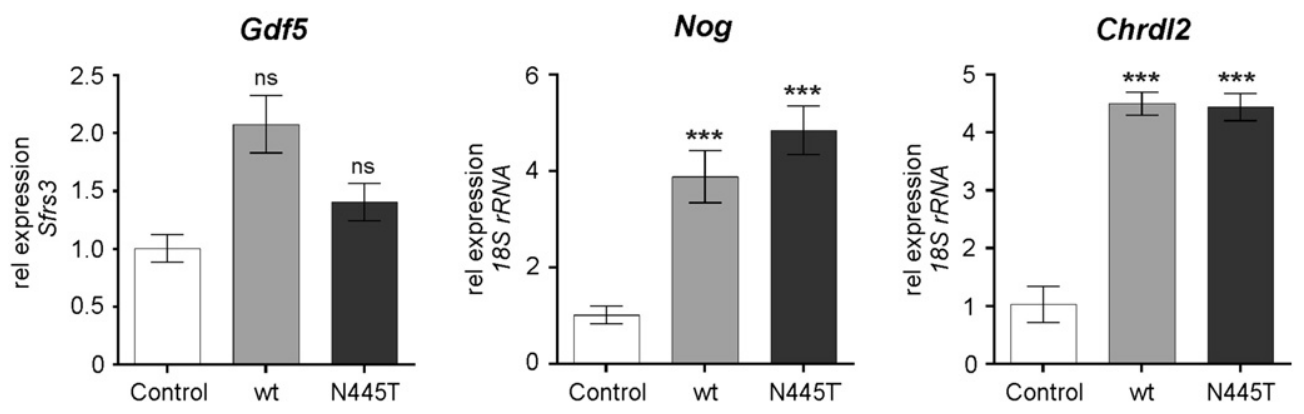

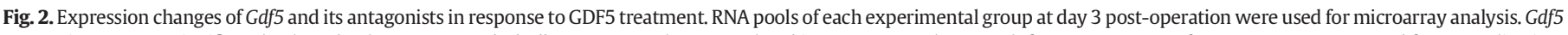

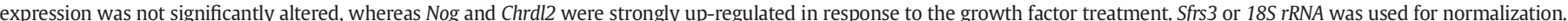

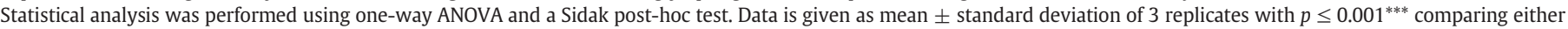
$\mathrm{GDF}^{\mathrm{wt}}$ or GDF5 ${ }^{\mathrm{N} 445 \mathrm{~T}}$ with the control.

heatmap. By visual inspection we subdivided the heatmap into 7 major gene regulatory clusters (Fig. 3A).

Clusters 1-6 are relatively homogenous, whereas cluster 7 that includes about one fifth of all 517 high-variance genes represents a rather heterogenic group of genes. The characteristic expression pattern of each cluster is shown as a line separated according to the experimental groups connecting the normalized mean values and SD of all genes within a cluster corresponding to the indicated time point (Fig. 3B). In line with the visual impression, cluster 7 shows the longest whiskers in these line plots. For functional annotation of the genes in each cluster, GO analysis was performed and the top GO terms are listed on the right side of each cluster (Fig. 3C).

In accordance with the $\mathrm{GO}$ analysis of all high-variance genes, genes localized in cluster 3 are mainly annotated to 'ossification' (8/50), 'cell adhesion' (10/50), and 'tissue remodeling' (5/50) and although GO analysis of cluster 5 revealed no significance, $6 / 40$ genes are likewise annotated to 'ossification' and 2/40 to 'osteoblast development'. In cluster 4 we identified 12/35 genes related to 'muscle system process', which are believed to be responsible for the creation of contractile forces that are needed in bony tissues for stiffening of the ECM prior to mineralization. Cluster 7 contains genes with no homogenous expression values and revealed general terms, which are not specific for a distinct cellular program, but related to general processes relevant in the setting of bone healing.

GDF5 ${ }^{N 445 T}$ treatment resulted in faster downregulation of inflammatory marker genes

Among the clusters, clusters 1 and 2 comprise genes that are related to immunological processes. In cluster 1, GO terms like 'cytokine production' and 'immune response' are over represented, which are fundamental processes during the early phase of healing, when the repair cascade is initiated. Within this cluster, centrally important genes such as Interleukin-1 Beta (Il1b), Interleukin-6 (Il6), Chemokine ( $\mathrm{C}-\mathrm{X}-\mathrm{C}$ motif) ligand 2 ( $\mathrm{Cxcl} 2$ ), and Chemokine ( $\mathrm{C}-\mathrm{C}$ motif) ligand 3 ( $\mathrm{Ccl} 3$ ) show highest expression at day 3. Interestingly, in the GDF5 ${ }^{\mathrm{N} 445 \mathrm{~T}}$-stimulated group these inflammatory genes show the most rapid decrease and overall lowest expression levels at day 7 (Figs. 3B, C). Q-PCR analysis for $I l 1 b$ and $C c l 3$ confirmed a markedly faster downregulation in GDF5 ${ }^{\mathrm{N} 445 \mathrm{~T}}$ - than in $\mathrm{GDF} 5^{\mathrm{wt}}$-treated defects (Fig. 4).

Cluster 2 includes many genes related to the immune system showing highest expression levels at day 14. In this cluster 18/84 genes are annotated to 'immune system process' and 7/84 genes are annotated to 'leukocyte differentiation'. In the GDF5 ${ }^{\mathrm{N} 445 \mathrm{~T}}$-treated samples, genes in cluster 2 are expressed on a rather stable level at days 3,5, and 7, whereas their expression in the control and GDF5 ${ }^{\mathrm{wt}}$-treated group shows more fluctuations (Figs. 3B, C).
GDF5 $5^{\text {N4454T }}$ treatment resulted in early up-regulation of chondrogenic marker genes

Characteristic cartilaginous matrix genes including Collagen type 2 alpha 1 (Col2a1), Matrillin-3 (Matn3), Aggrecan (Acan), and Cartilage oligomeric protein (Comp) were identified in cluster 6 , and upregulated as compared to the control group at days 7 and 14. Almost half of the genes in this cluster are annotated to terms related to development such as 'cellular development' and 'developmental process'. Here, the GDF5 ${ }^{\text {N445T }}$-treated group showed a more rapid up-regulation of these genes especially at days 5 and 7 (Figs 3B, C). Similarly, the extracellular matrix (ECM) molecule Acan and SRY-Box 9 (Sox9), one of the earliest marker of cells undergoing condensation, were strongly affected by the GDF5-treatments and showed elevated expression already at day 5 post-operation (Fig. 5 ). Notably, the GDF5 ${ }^{\mathrm{N} 445 \mathrm{~T}}$-induced increase was transient and a decline in expression levels was observed in control and $\mathrm{GDF}^{\mathrm{N} 445 \mathrm{~T}}$-treated defects but not in GDF5 ${ }^{\mathrm{wt}}$-treated defects during the time period of analysis. The same trend was observed for Col2a1 and Comp (Fig. 5). These findings further support the notion, that GDF5 $^{\mathrm{N} 445 \mathrm{~T}}$ accelerates the endochondral bone healing process in comparison to GDF5 ${ }^{\mathrm{wt}}$.

\section{Discussion}

In this study, we report a side-by-side comparison of GDF5 ${ }^{\mathrm{wt}}$ - and $\mathrm{GDF}^{\mathrm{N} 445 \mathrm{~T}}$-treated bone defects in comparison to control defects. The data indicate improved bony callus formation and regeneration upon GDF5 $^{\mathrm{N} 445 \mathrm{~T}}$ treatment in comparison to control or GDF5 ${ }^{\mathrm{wt}}$ treatment. Previous work has shown that the N445T point mutation destabilizes the interaction with the extracellular antagonist NOG, resulting in a markedly increased GDF5 signaling output, which led in vitro to a strong chondrogenic effect on primary mesenchymal limb bud cells [23]. In light of these results, we were encouraged to evaluate the regenerative capacity of GDF5 ${ }^{\mathrm{N} 445 \mathrm{~T}}$ in vivo using a well-established rat critical-sized defect model [27] that has already been successfully used for the evaluation of bone repair stimulation strategies $[26,36]$. In accordance with previous studies, we observed that osteotomy sites treated with GDF5 $^{\text {wt }}$ heal via classical endochondral ossification [36]. Our data indicate that also GDF5 ${ }^{\mathrm{N} 445 \mathrm{~T}}$-stimulated regeneration follows the same general pathway. However, the molecular events appear to occur faster after local application of the GDF5 ${ }^{\mathrm{N} 445 \mathrm{~T}}$ variant as compared to $\mathrm{GDF}^{\mathrm{wt}}$, in line with the accelerated bone defect healing. Molecular characterization of the healing phase indicates an early local upregulation of BMP antagonists, such as Nog and Chrdl2 in GDF5 ${ }^{\text {wt }}$ - and GDF5 $^{\text {N445T }}$-treated rats. Whereas it is well established that NOG directly regulates GDF5 activity, there is only one study addressing the interaction between CHRDL2 and GDF5 [37]. Although we could not confirm that CHRDL2 inhibits GDF5 ${ }^{\mathrm{wt}}$ activity using an ALP assay, the signaling activity of GDF5 ${ }^{\mathrm{N} 445 \mathrm{~T}}$ might have been retained for a longer period of 


\section{A) Hierarchical clustering}

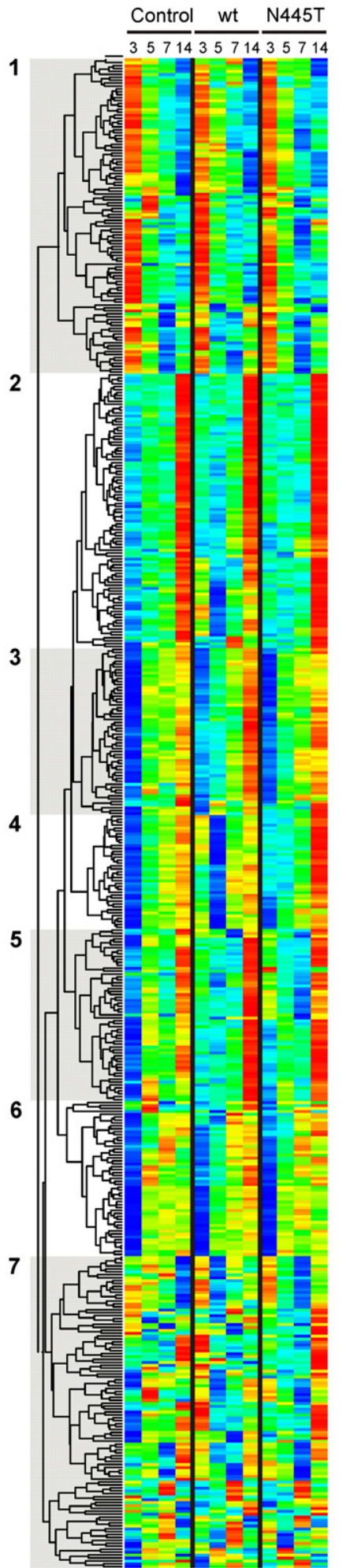

\section{B) Cluster expression dynamics}

\author{
Control wt N445T
}
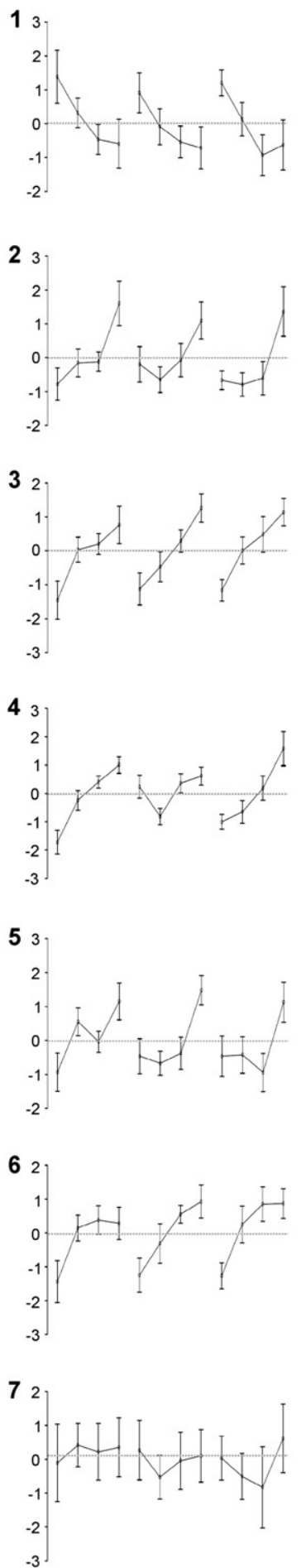

\section{C) Cluster Gene Ontology analysis}

Pop. Study p

term term adjuste

\begin{tabular}{lccc} 
Cluster 1, Study total: 99 & & & \\
\hline cytokine production & 241 & 14 & $1,4 \mathrm{E}-06$ \\
regulation of cytokine production & 211 & 14 & $7,9 \mathrm{E}-05$ \\
nitric oxide metabolic process & 43 & 6 & $1,2 \mathrm{E}-04$ \\
immune response & 543 & 19 & $1,2 \mathrm{E}-04$ \\
$\begin{array}{l}\text { regulation of nitric oxide } \\
\text { biosynthetic process }\end{array}$ & 32 & 5 & $1,2 \mathrm{E}-04$ \\
\end{tabular}

biosynthetic process

Cluster 2, Study total: $\mathbf{8 4}$

immune system process

$\begin{array}{llll} & 143 & 6 & 0,025\end{array}$

$200 \quad 7 \quad 0,105$

Cluster 3, Study total: $\mathbf{5 0}$

\begin{tabular}{lccc}
\hline ossification & 190 & 8 & 0,005 \\
biological adhesion & 505 & 10 & 0,010 \\
cell adhesion & 505 & 10 & 0,010 \\
tissue remodeling & 91 & 5 & 0,028 \\
locomotion & 615 & 10 & 0,028
\end{tabular}

Cluster 4, Study total: 35 muscle system process $\begin{array}{llll}\text { muscle tissue morphogenesis } & 35 & 4 & 2,7 \mathrm{E}-03\end{array}$

\section{Cluster $\mathbf{5}$, Study total: $\mathbf{4 0}$}

ossification

regulation of biomine

tissue development

$\begin{array}{llll}\text { osteoblast development } & 15 & 2 & 0,491\end{array}$

Cluster 6, Study total: $\mathbf{4 9}$

\begin{tabular}{lccc}
\hline cell adhesion & 505 & 8 & 0,069 \\
cellular developmental process & 1721 & 15 & 0,069 \\
developmental process & 2836 & 21 & 0,069 \\
biological adhesion & 505 & 8 & 0,089 \\
skeletal system development & 219 & 7 & 0,211
\end{tabular}

Cluster 7, Study total: 82

multi-organism process

response to biotic stimulus

response to stress

$\begin{array}{lccc}\text { defense response to fungus } & 9 & 4 & 1,3 \mathrm{E}-02\end{array}$

negative regulation of

multi-organism process

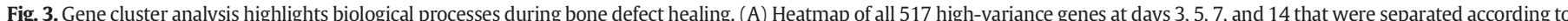

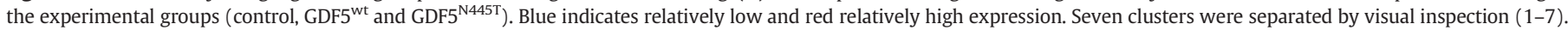

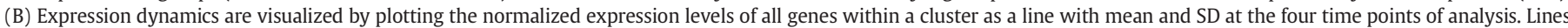

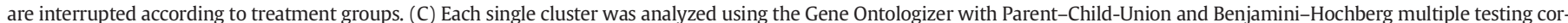

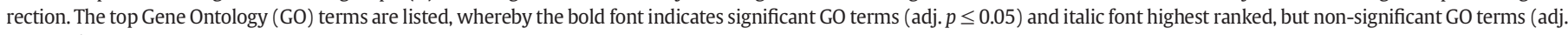
$\mathrm{p} \geq 0.05$ ). 

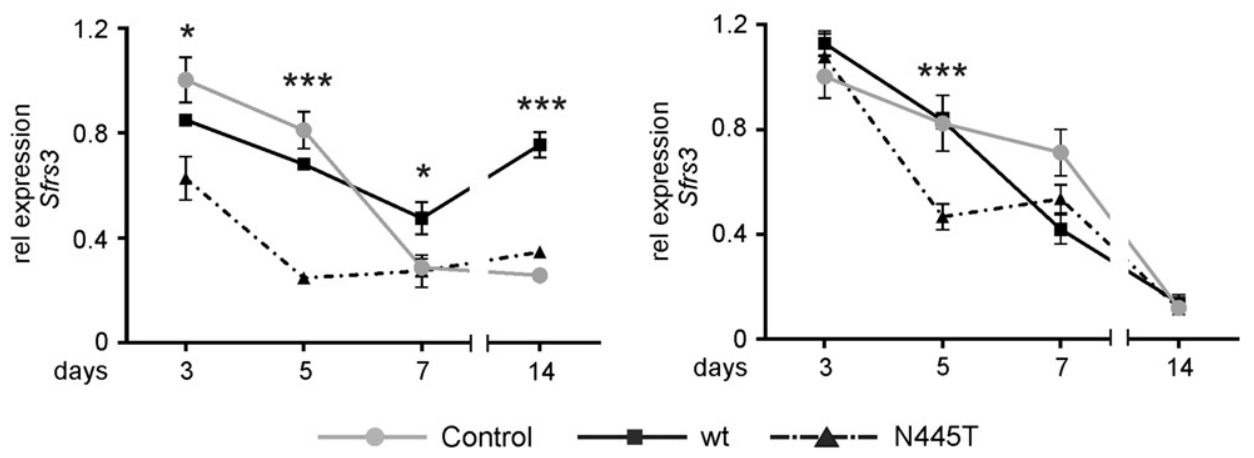

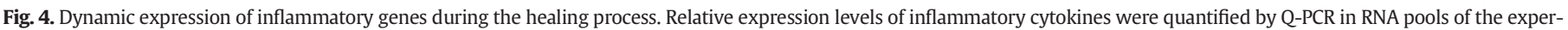

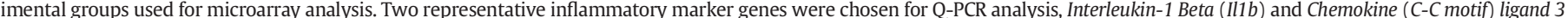

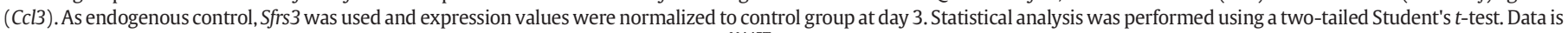
given as mean \pm SD of 3 replicates with $p \leq 0.05^{*}$, and $p \leq 0.001^{* * *}$ comparing GDF5 ${ }^{\mathrm{N} 445 \mathrm{~T}}$ - with GDF5 ${ }^{\mathrm{wt}}$-treated samples.

time due to its NOG insensitivity. The global expression analysis of the early healing phase indicates a distinct temporal difference in inflammatory gene expression between the GDF5 ${ }^{\mathrm{wt}}$ - and GDF5 ${ }^{\mathrm{N} 445 \mathrm{~T}}$-treated samples. Inflammation is the initial event during bone healing, in which a hematoma is formed due to accumulating blood originating from the damaged bone ends [38]. Recent studies highlight that the immune cell function is regulated during bone healing and that the modulation of immune cell function is a promising approach to improve regeneration [39-41]. In contrast to GDF5 ${ }^{\mathrm{wt}}$, we observed that the pro-inflammatory marker gene $I l 1 b$ and the inflammatory chemokine $\mathrm{CCl} 3$ were more rapidly declining after the GDF5 ${ }^{\mathrm{N} 445 \mathrm{~T}}$ stimulus suggesting that these fracture sites have entered an advanced level within the healing cascade. Furthermore, the decline in inflammatory gene expression was strongly linked with an early rise in cartilage-specific matrix genes. Especially the expression of type II collagen, like Col2a1 by chondrogenic cells is an indicator for endochondral ossification accompanying cartilaginous matrix production and preceding soft callus formation $[42,43]$.

As cell volume increases, we found the ECM molecule Acan to be strongly up-regulated, likely needed to control the osmotic pressure [44]. Ascending expression levels as response to the terminating inflammatory signal in GDF5 ${ }^{\mathrm{N} 445}$-treated rats were observed in case of Sox9, one of the central transcription factors regulating chondrocyte differentiation [45], and Comp, another key ECM component of functional cartilage [46]. An attenuated gene expression of Sox 9 and Acan in GDF5 ${ }^{\mathrm{N} 445 \mathrm{~T}}$ treated rats was observed between days 7 and 14 post-operation
Sox 9
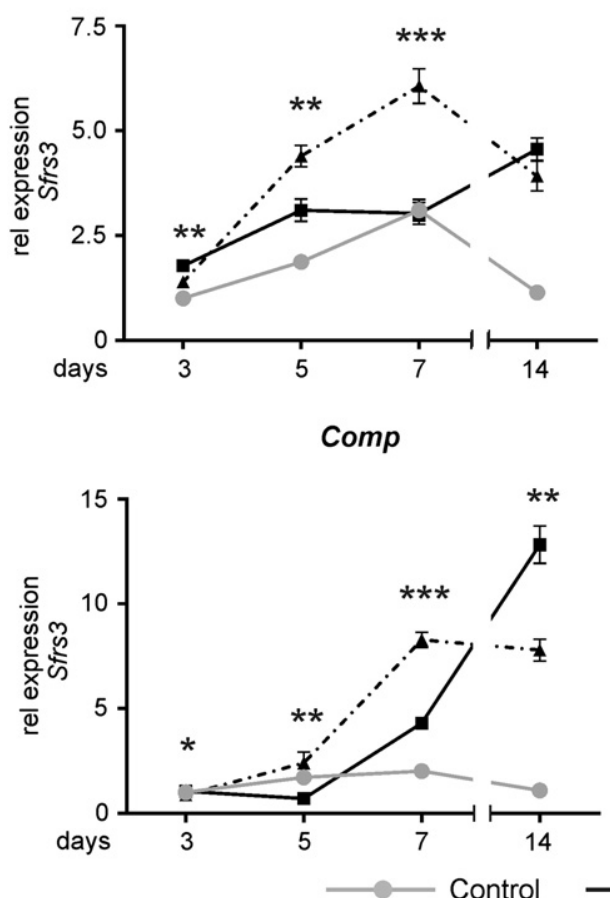
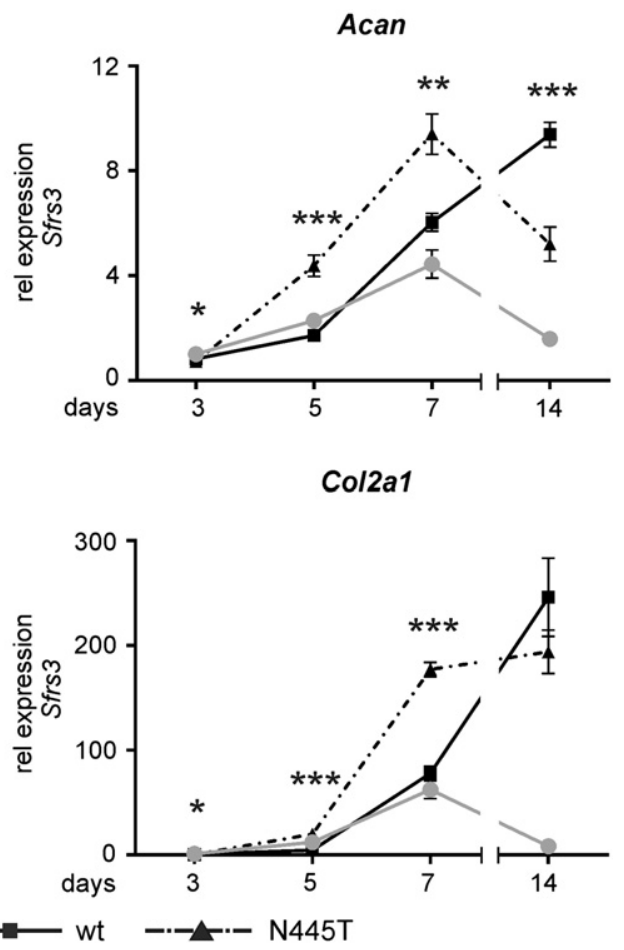

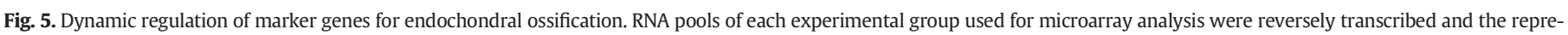

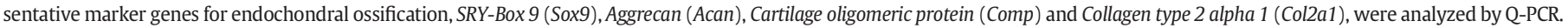

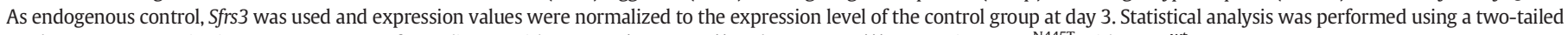
Student's $t$-test. Data is given as mean \pm SD of 3 replicates with $p \leq 0.05^{*}, p \leq 0.01^{* *}$ and,$p \leq 0.001^{* * *}$ comparing GDF5 $^{\mathrm{N} 445 \mathrm{~T}}$ with GDF5 ${ }^{\mathrm{wt}}$. 
suggesting that cell differentiation is triggered earlier into the osteogenic lineage as compared to GDF5 ${ }^{\mathrm{wt}}$ stimulated osteotomy sites.

The principle of rendering growth factors more potent through mutations has been previously addressed, e.g., by introducing two mutations into GDF5 yielding the bioengineered GDF5 $5^{\mathrm{M} 453 \mathrm{~V}}$, M456V (BB-1) molecule $[47,48]$. BB-1 shows an altered receptor binding characteristic converting GDF5 into a BMP2-like molecule. Other attempts included the introduction of the BMP2 heparin binding sites N-terminally of GDF5 (B2GDF5); however, the in vivo healing outcome using this variant was indistinguishable from GDF5 ${ }^{\mathrm{wt}}$ treatment [49]. A slightly better result was obtained using the monomeric variant of GDF5, GDF5 ${ }^{\mathrm{C} 456 \mathrm{~A}}$, where the healing capacity was improved as compared to B2GDF5 [50].

An advantage of GDF5 ${ }^{\mathrm{N} 445 \mathrm{~T}}$ might be the feature that only a single amino acid within the NOG binding interface is replaced retaining an unaffected signal transduction pathway and thus causing comparable effects on the gene expression pattern as compared to GDF5 ${ }^{\mathrm{wt}}$. As GDF5 $^{\text {N445T }}$ is a naturally occurring mutation, it has already proven its markedly enhanced signaling capacity leading to bony fusions in the patients. Moreover, there are no severe side-effects reported in SYNS2 patients such as heterotopic ossification or increased cancer risk. In relation to its therapeutic potential, it appears as if critical steps during the bone defect repair become accelerated and strengthened by GDF5 $^{\mathrm{N} 445 \mathrm{~T}}$ as compared to its wild type counterpart, likely due to the avoidance of NOG-mediated inhibition. Such a bypassing of the negative feedback regulation was also reported by Song and colleagues [51] by transferring a key residue of BMP6 responsible for the NOG resistance into the corresponding position of BMP2 and BMP7. It can be assumed that the strategy employed in our study can also be adapted to other growth factors for rendering them less sensitive to endogenous feedback control and downregulation by increased antagonist expression.

As it is known that growth factors have different receptor binding preferences and therefore also different regenerative capacities, a tool box of highly potent growth factors can be generated for various therapeutic applications by transferring the same principle to other growth factors to render them more potent by introducing a homologous mutation within the NOG binding site.

\section{Conclusion}

Our study supports the concept of improving growth factor signaling and specificity for therapeutic use by modifying key residues involved in antagonist interaction. Applying this strategy to other growth factors will further enlarge the toolbox available to clinicians for personalized therapeutic use. We are convinced that a great potential for improving the signaling capacity of biomolecules lies hidden in the naturally occurring mutations identified in rare diseases. This strategy has several advantages, as the critical residues for a given biological process are identified during molecular diagnosis, the major effects are highlighted by the symptoms, and some knowledge on the biological safety and side-effects of the variants can be deduced from a thorough analysis of potential co-morbidities observed in the patients.

\section{Acknowledgments}

We thank Mario Thiele and Cornelia Schneider for microCT support, Ute Ungethüm for Affymetrix chip hybridizations and Lutz Schomburg for help with RNA isolation and for critical remarks on the manuscript.

ED and CS are members of the DFG funded Berlin-Brandenburg School for Regenerative Therapies GSC 203. This work was supported by the Berlin-Brandenburg Center for Regenerative Therapies (BCRT) (1315848A) and by the Deutsche Forschungsgemeinschaft (DFG) grant number SFB 760 (to JL, SM, GND, PS), MU 880/11-01 (to CEO, SM), SE 1778/1 (to PS), and DU 298/15-1 (to GND).

\section{Conflict of interest statement}

Frank Plöger is an employee of the company Biopharm GmbH as indicated in the affiliation. Biopharm $\mathrm{GmbH}$ is a $100 \%$ privately owned company. The Co-authors Frank Plöger and Stefan Mundlos have filed a patent on the GDF5 variant N445T (PCT/EP2006/001966). All other authors have declared that no competing interests exist.

\section{Appendix A. Supplementary data}

Supplementary data to this article can be found online at http://dx. doi.org/10.1016/j.bone.2014.12.017.

\section{References}

[1] Knight MN, Hankenson KD. Mesenchymal stem cells in bone regeneration. Adv Wound Care (New Rochelle) 2013;2:306-16.

[2] Coleman CM, Vaughan EE, Browe DC, Mooney E, Howard L, Barry F. Growth differentiation factor-5 enhances in vitro mesenchymal stromal cell chondrogenesis and hypertrophy. Stem Cells Dev 2013;22:1968-76.

[3] Hotten GC, Matsumoto T, Kimura M, Bechtold RF, Kron R, Ohara T, et al. Recombinant human growth/differentiation factor 5 stimulates mesenchyme aggregation and chondrogenesis responsible for the skeletal development of limbs. Growth Factors 1996;13:65-74.

[4] Rountree RB, Schoor M, Chen H, Marks ME, Harley V, Mishina Y, et al. BMP receptor signaling is required for postnatal maintenance of articular cartilage. PLoS Biol 2004; 2:e355.

[5] Storm EE, Huynh TV, Copeland NG, Jenkins NA, Kingsley DM, Lee SJ. Limb alterations in brachypodism mice due to mutations in a new member of the TGF betasuperfamily. Nature 1994;368:639-43.

[6] Luyten FP. Cartilage-derived morphogenetic protein-1. Int J Biochem Cell Biol 1997; 29:1241-4.

[7] Schwarz F, Rothamel D, Herten M, Ferrari D, Sager M, Becker J. Lateral ridge augmentation using particulated or block bone substitutes biocoated with rhGDF-5 and rhBMP-2: an immunohistochemical study in dogs. Clin Oral Implants Res 2008;19: 642-52.

[8] Weng D, Poehling S, Pippig S, Bell M, Richter EJ, Zuhr O, et al. The effects of recombinant human growth/differentiation factor-5 (rhGDF-5) on bone regeneration around titanium dental implants in barrier membrane-protected defects: a pilot study in the mandible of beagle dogs. Int J Oral Maxillofac Implants 2009;24:31-7.

[9] Yoshimoto T, Yamamoto M, Kadomatsu H, Sakoda K, Yonamine Y, Izumi Y. Recombinant human growth/differentiation factor-5 (rhGDF-5) induced bone formation in murine calvariae. J Periodontal Res 2006;41:140-7.

[10] Poehling S, Pippig SD, Hellerbrand K, Siedler M, Schutz A, Dony C. Superior effect of MD05, beta-tricalcium phosphate coated with recombinant human growth/ differentiation factor-5, compared to conventional bone substitutes in the rat calvarial defect model. J Periodontol 2006;77:1582-90.

[11] Chujo T, An HS, Akeda K, Miyamoto K, Muehleman C, Attawia M, et al. Effects of growth differentiation factor-5 on the intervertebral disc-in vitro bovine study and in vivo rabbit disc degeneration model study. Spine (Phila Pa 1976) 2006;31: 2909-17.

[12] Magit DP, Maak T, Trioano N, Raphael B, Hamouria Q, Polzhofer G, et al. Healos/recombinant human growth and differentiation factor-5 induces posterolateral lumbar fusion in a New Zealand white rabbit model. Spine (Phila Pa 1976) 2006;31: 2180-8.

[13] Spiro RC, Thompson AY, Poser JW. Spinal fusion with recombinant human growth and differentiation factor-5 combined with a mineralized collagen matrix. Anat Rec 2001;263:388-95.

[14] Spiro RC, Liu L, Heidaran MA, Thompson AY, Ng CK, Pohl J, et al. Inductive activity of recombinant human growth and differentiation factor-5. Biochem Soc Trans 2000; 28:362-8.

[15] Chhabra A, Zijerdi D, Zhang J, Kline A, Balian G, Hurwitz S. BMP-14 deficiency inhibits long bone fracture healing: a biochemical, histologic, and radiographic assessment. J Orthop Trauma 2005;19:629-34.

[16] Katayama R, Wakitani S, Tsumaki N, Morita Y, Matsushita I, Gejo R, et al. Repair of articular cartilage defects in rabbits using CDMP1 gene-transfected autologous mesenchymal cells derived from bone marrow. Rheumatology (Oxford) 2004;43:980-5.

[17] Wolfman NM, Hattersley G, Cox K, Celeste AJ, Nelson R, Yamaji N, et al. Ectopic induction of tendon and ligament in rats by growth and differentiation factors 5, 6, and 7, members of the TGF-beta gene family. J Clin Invest 1997;100:321-30.

[18] Forslund C, Rueger D, Aspenberg P. A comparative dose-response study of cartilagederived morphogenetic protein (CDMP) $-1,-2$ and -3 for tendon healing in rats. J Orthop Res 2003;21:617-21.

[19] Bolt P, Clerk AN, Luu HH, Kang Q Kummer JL, Deng ZL, et al. BMP-14 gene therapy increases tendon tensile strength in a rat model of Achilles tendon injury. J Bone Joint Surg Am 2007;89:1315-20.

[20] Moore YR, Dickinson DP, Wikesjo UM. Growth/differentiation factor-5: a candidate therapeutic agent for periodontal regeneration? A review of pre-clinical data. J Clin Periodontol 2010;37:288-98.

[21] Dawson K, Seeman P, Sebald E, King L, Edwards M, Williams 3rd J, et al. GDF5 is a second locus for multiple-synostosis syndrome. Am J Hum Genet 2006;78:708-12. 
[22] Schwaerzer GK, Hiepen C, Schrewe H, Nickel J, Ploeger F, Sebald W, et al. New insights into the molecular mechanism of multiple synostoses syndrome (SYNS): mutation within the GDF5 knuckle epitope causes noggin-resistance. J Bone Miner Res 2012;27:429-42.

[23] Seemann P, Brehm A, Konig J, Reissner C, Stricker S, Kuss P, et al. Mutations in GDF5 reveal a key residue mediating BMP inhibition by NOGGIN. PLoS Genet 2009;5: e1000747.

[24] Seemann P, Schwappacher R, Kjaer KW, Krakow D, Lehmann K, Dawson K, et al. Activating and deactivating mutations in the receptor interaction site of GDF5 cause symphalangism or brachydactyly type A2. J Clin Invest 2005;115:2373-81.

[25] Degenkolbe E, Konig J, Zimmer J, Walther M, Reissner C, Nickel J, et al. A GDF5 point mutation strikes twice-causing BDA1 and SYNS2. PLoS Genet 2013;9:e1003846.

[26] Schwarz C, Wulsten D, Ellinghaus A, Lienau J, Willie BM, Duda GN. Mechanical load modulates the stimulatory effect of BMP2 in a rat nonunion model. Tissue Eng Part A 2013;19:247-54.

[27] Mehta M, Schell H, Schwarz C, Peters A, Schmidt-Bleek K, Ellinghaus A, et al. A 5-mm femoral defect in female but not in male rats leads to a reproducible atrophic nonunion. Arch Orthop Trauma Surg 2011;131:121-9.

[28] Bouxsein ML, Boyd SK, Christiansen BA, Guldberg RE, Jepsen KJ, Muller R. Guidelines for assessment of bone microstructure in rodents using micro-computed tomography. J Bone Miner Res 2010;25:1468-86.

[29] Kallai I, Mizrahi O, Tawackoli W, Gazit Z, Pelled G, Gazit D. Microcomputed tomography-based structural analysis of various bone tissue regeneration models. Nat Protoc 2011;6:105-10.

[30] Morgan EF, Mason ZD, Chien KB, Pfeiffer AJ, Barnes GL, Einhorn TA, et al. Microcomputed tomography assessment of fracture healing: relationships among callus structure, composition, and mechanical function. Bone 2009;44:335-44.

[31] Bengtsson KS H, Bullard J, Hansen K. A generic framework in R for analyzing small to very large Affymetrix data sets in bounded memory. Tech Report \#745. Berkeley: Department of Statistics, University of California; February 2008.

[32] Kauffmann A, Gentleman R, Huber W. arrayQualityMetrics-a bioconductor package for quality assessment of microarray data. Bioinformatics 2009;25:415-6.

[33] Bauer S, Gagneur J, Robinson PN. GOing Bayesian: model-based gene set analysis of genome-scale data. Nucleic Acids Res 2010;38:3523-32.

[34] Ashburner M, Ball CA, Blake JA, Botstein D, Butler H, Cherry JM, et al. Gene ontology: tool for the unification of biology. The Gene Ontology Consortium. Nat Genet 2000; 25:25-9.

[35] Bauer S, Grossmann S, Vingron M, Robinson PN. Ontologizer 2.0-a multifunctional tool for GO term enrichment analysis and data exploration. Bioinformatics 2008; 24:1650-1.

[36] Wulsten D, Glatt V, Ellinghaus A, Schmidt-Bleek K, Petersen A, Schell H, et al. Time kinetics of bone defect healing in response to BMP-2 and GDF-5 characterised by in vivo biomechanics. Eur Cell Mater 2011;21:177-92.

[37] Nakayama N, Han CY, Cam L, Lee JI, Pretorius J, Fisher S, et al. A novel chordin-like BMP inhibitor, CHL2, expressed preferentially in chondrocytes of developing cartilage and osteoarthritic joint cartilage. Development 2004;131:229-40.

[38] Kolar P, Schmidt-Bleek K, Schell H, Gaber T, Toben D, Schmidmaier G, et al. The early fracture hematoma and its potential role in fracture healing. Tissue Eng Part B Rev 2010;16:427-34.

[39] Reinke S, Geissler S, Taylor WR, Schmidt-Bleek K, Juelke K, Schwachmeyer V, et al Terminally differentiated CD8 $(+)$ T cells negatively affect bone regeneration in humans. Sci Transl Med 2013;5:177ra36.

[40] Schmidt-Bleek K, Schell H, Lienau J, Schulz N, Hoff P, Pfaff M, et al. Initial immune reaction and angiogenesis in bone healing. J Tissue Eng Regen Med 2014;8:120-30.

[41] Schmidt-Bleek K, Schell H, Schulz N, Hoff P, Perka C, Buttgereit F, et al. Inflammatory phase of bone healing initiates the regenerative healing cascade. Cell Tissue Res 2012;347:567-73.

[42] Allori AC, Sailon AM, Warren SM. Biological basis of bone formation, remodeling, and repair-part II: extracellular matrix. Tissue Eng Part B Rev 2008;14:275-83.
[43] Tsiridis E, Upadhyay N, Giannoudis P. Molecular aspects of fracture healing: which are the important molecules? Injury 2007;38(Suppl. 1):S11-25.

[44] Chandran PL, Horkay F. Aggrecan, an unusual polyelectrolyte: review of solution behavior and physiological implications. Acta Biomater 2012;8:3-12.

[45] Ng L], Wheatley S, Muscat GE, Conway-Campbell J, Bowles J, Wright E, et al. SOX9 binds DNA, activates transcription, and coexpresses with type II collagen during chondrogenesis in the mouse. Dev Biol 1997;183:108-21.

[46] Motaung SC, Di Cesare PE, Reddi AH. Differential response of cartilage oligomeric matrix protein (COMP) to morphogens of bone morphogenetic protein/ transforming growth factor-beta family in the surface, middle and deep zones of articular cartilage. J Tissue Eng Regen Med 2011;5:e87-96.

[47] Kasten P, Beyen I, Bormann D, Luginbuhl R, Ploger F, Richter W. The effect of two point mutations in GDF-5 on ectopic bone formation in a beta-tricalciumphosphate scaffold. Biomaterials 2010;31:3878-84.

[48] Kleinschmidt K, Ploeger F, Nickel J, Glockenmeier J, Kunz P, Richter W. Enhanced reconstruction of long bone architecture by a growth factor mutant combining positive features of GDF-5 and BMP-2. Biomaterials 2013;34:5926-36.

[49] Depprich R, Handschel J, Sebald W, Kubler NR, Wurzler KK. Comparison of the osteogenic activity of bone morphogenetic protein (BMP) mutants. Mund Kiefer Gesichtschir 2005;9:363-8.

[50] Dupoirieux L, Pohl J, Hanke M, Pourquier D. A preliminary report on the effect of dimeric rhGDF-5 and its monomeric form rhGDF-5C465A on bone healing of rat cranial defects. J Craniomaxillofac Surg 2009;37:30-5.

[51] Song K, Krause C, Shi S, Patterson M, Suto R, Grgurevic L, et al. Identification of a key residue mediating bone morphogenetic protein (BMP)-6 resistance to noggin inhibition allows for engineered BMPs with superior agonist activity. J Biol Chem 2010;285:12169-80.

\section{Glossary}

Acan: Aggrecan

$B M a$ : bone marrow

$B M P$ : Bone Morphogenetic Protein

BMPR1A: BMP receptor type $1 \mathrm{~A}$

BMPR1B: BMP receptor type 1B

BMPR2: BMP receptor type 2

$B V$ : bone volume

Ccl3: chemokine ( $\mathrm{C}-\mathrm{C}$ motif) ligand 3

CDMP1: cartilage-derived

Chrdl2: Chordin-like 2

$\mathrm{Co}$ : connective tissue

Col2a1: collagen, type II, alpha 1

Comp: cartilage oligomeric matrix protein

CT: computer tomography

GDF: Growth and Differentiation Factor

GO: Gene Ontology

$H A$ : hydroxyapatite

$I l 1 b$ : interleukin 1 beta

Mc: muscle

NOG: Noggin

$Q-P C R$ : quantitative polymerase chain reaction

Sox9: SRY (sex determining region Y)-box 9

SYNS2: multiple synostoses syndrome 2

TMC: tissue mineral content

$T M D$ : tissue mineral density

TV: total volume

VOI: volume of interest

$\mathrm{Wb}$ : woven bone

$w t$ : wild type 